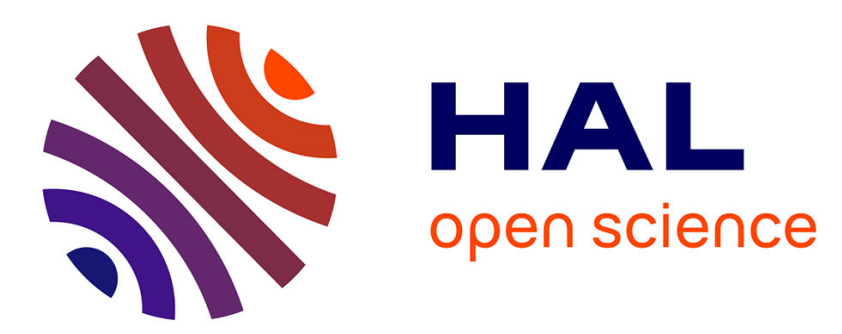

\title{
Evidence of growing spatial correlations at the glass transition from nonlinear susceptibility measurements
}

\author{
C. Brun, C. Crauste-Thibierge, D. L'Hôte, F. Ladieu, G. Biroli, J.-P.
} Bouchaud

\section{- To cite this version:}

C. Brun, C. Crauste-Thibierge, D. L'Hôte, F. Ladieu, G. Biroli, et al.. Evidence of growing spatial correlations at the glass transition from nonlinear susceptibility measurements. physica status solidi (c), 2011, 8 (11-12), pp.3147 - 3150. 10.1002/pssc.201000718 . cea-01396584

\section{HAL Id: cea-01396584 https://hal-cea.archives-ouvertes.fr/cea-01396584}

Submitted on 14 Nov 2016

HAL is a multi-disciplinary open access archive for the deposit and dissemination of scientific research documents, whether they are published or not. The documents may come from teaching and research institutions in France or abroad, or from public or private research centers.
L'archive ouverte pluridisciplinaire HAL, est destinée au dépôt et à la diffusion de documents scientifiques de niveau recherche, publiés ou non, émanant des établissements d'enseignement et de recherche français ou étrangers, des laboratoires publics ou privés. 


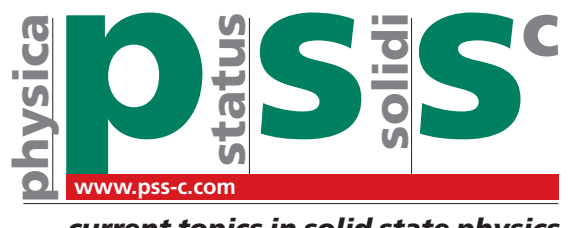

\title{
Evidence of growing spatial correlations at the glass transition from nonlinear susceptibility measurements
}

\author{
C. Brun ${ }^{*}, 1$, C. Crauste-Thibierge ${ }^{2}$, D. L'Hôte ${ }^{1}$, F. Ladieu ${ }^{1}$, G. Biroli ${ }^{3}$, and J.-P. Bouchaud ${ }^{4}$ \\ ${ }^{1}$ SPEC (CNRS URA 2464), DSM/IRAMIS CEA Saclay, Bat. 772, 91191 Gif-sur-Yvette France \\ ${ }^{2}$ LLB (CNRS UMR 12), DSM/IRAMIS CEA Saclay, Bat. 563, 91191 Gif-sur-Yvette France \\ ${ }^{3}$ Institut de Physique Théorique, CEA (CNRS URA 2306), 91191 Gif-sur-Yvette, France \\ ${ }^{4}$ Science \& Finance, Capital Fund Management, 6, Bd. Haussmann, 75009 Paris, France
}

Received 20 July 2010, revised 8 November 2010, accepted 9 November 2010

Published online 9 March 2011

Keywords glasses, nonlinear, susceptibility, dielectric

${ }^{*}$ Corresponding author: e-mail coralie.brun@cea.fr, Phone: +33 16 9087538, Fax: +33 169088786

We have measured the ac nonlinear dielectric response $\chi_{3}(\omega, T)$ of an archetypical glassformer (glycerol) just above its glass transition temperature $T_{g} \sim 190 \mathrm{~K}$. Our measurements were performed at temperatures between $T_{g}+4 \mathrm{~K}$ and $T_{g}+35 \mathrm{~K}$, which corresponds to 5 decades of variation of the relaxation time $\tau(T)$ of the liquid. We find that $\chi_{3}(\omega, T)$ is peaked for $\omega \tau \sim 0.2$ and that the height of the peak grows as one approaches $T_{g}$. A powerlaw in frequency beyond the peak of $\chi_{3}$ is observed and $\chi_{3}(\omega, T)$ displays scaling as a function of $\omega \tau$.
These features correspond to the main predictions of some recent theoretical works relating the nonlinear susceptibility of supercooled liquids to the average number $N_{\text {corr }}$ of dynamically correlated molecules. We thus interpret the experimental increase of the peak of nonlinear susceptibility when $T$ decreases, as an increase of $N_{\text {corr }}$ when $T \rightarrow T_{g}$. This strongly reinforces the collective picture of the dynamics of supercooled liquids close to the glass transition. Finally, we discuss some heating effects and show that they do not affect significantly our conclusions.

(๖ 2011 WILEY-VCH Verlag GmbH \& Co. KGaA, Weinheim

1 Introduction Upon fast enough cooling, most liquids avoid crystallization and enter in a supercooled state. In this supercooled state, the "viscous slowing down" phenomenon arises [1], i.e. the viscosity $\eta$ of supercooled liquids increases extremely fast when the temperature $T$ is decreased, this increase being even faster than Arrhenius in the so-called fragile glasses. Below $T_{g}$ the viscosity is so high that the system is, in practice, a solid, the glass. This glass transition is a longstanding issue of condensed matter physics, since no direct structural signature has ever been detected around $T_{g}$, contrary to what happens at the crystallization transition [1]. Some progresses about the glass transition were made in the two past decades, where some experimental breakthroughs [2,3] as well as numer- ical works [6] established the heterogeneous nature of the dynamics of supercooled liquids. These progresses led to the important concept of "dynamical heterogeneities" [4, 5], according to which the relaxation events come from collective motions of groups of typically $N_{\text {corr }}$ molecules: some of these groups are faster than others, but none of them are permanent entities, they all evolve in time. It has been proposed that the huge increase of $\eta$ in supercooled liquids comes from the fact that this number $N_{\text {corr }}$ increases as $T$ decreases towards $T_{g}$. The variation of $N_{\text {corr }}$ with temperature thus became an important issue in the field, triggering new theoretical ideas [7-10]. One of these new ideas came from an analogy [10] with the well known spin glass physics where the nonlinear susceptibility di- 
verges at $T_{c}$, reflecting the long range amorphous order which sets in at the spin glass transition temperature $T_{c}$. Let us underline that no divergence occurs in the linear susceptibility of spin glasses due to the fact that two point correlation functions are "blind" to this amorphous long range order. This peculiar order is captured only by higher order correlations functions, and thus by their associated nonlinear responses (through fluctuation-dissipation relations). Based upon a generalized fluctuation-dissipation relation, it was proposed in Ref. [10], that a similar phenomenon occurs in supercooled liquids, with the key difference that the peak of the nonlinear response $\chi_{3}$ was predicted to occur at finite frequencies of order $1 / \tau(T)$ where $\tau(T)$ is the relaxation time of the supercooled liquid. For the first time, a susceptibility was directly related to the quantity $N_{\text {corr }}$ of interest, allowing to scrutinize its temperature dependence.

2 Experimental setup Motivated by the new prediction of Ref. [10], we have developed an experiment allowing to measure the nonlinear susceptibility of supercooled liquids by the detection of the third harmonics of the dielectric polarisation $P(t)$ [11]. The supercooled liquid fills the space between two thick metallic electrodes separated by a distance $e$, and is submitted to a field $E \cos (\omega t)$. As a result, a dielectric response $P_{1}$ arises at $\omega . P_{1}$ is dominated by the linear response $P_{l i n}$, corresponding to the linear susceptibility $\chi_{\text {lin }}^{\prime}(\omega)+i \chi_{\text {lin }}^{\prime \prime}(\omega)$ [11], and hereafter the frequency where $\chi_{\text {lin }}^{\prime \prime}$ is maximum defines the relaxation frequency $f_{\alpha}$, the latter being of order $1 / \tau$. Due to the nonlinear behavior of the system, $P(t)$ contains higher harmonics at frequencies $n \omega$, where $n$ is an odd integer for symmetry reasons (the even values of $n$ would be allowed if the field would contain a static part). The response $P_{3}$ at $3 \omega$ is thus the first signal which is directly proportional to the nonlinear properties of the liquid. In pratice, $P_{3}$ is dominated by the third harmonics susceptibility $\chi_{3}$ of the liquid (see $[11,12]$ for a precise definition of $\chi_{3}$ ). Due to the weakness of $P_{3} / P_{1}$, we have developed a special bridge technique, containing two samples of different thicknesses $e_{\text {thin }}<20 \mu \mathrm{m}$ and $e_{\text {thick }} \simeq 2 e_{\text {thin }}$. This allows to get rid of all the spurious $3 \omega$ signals coming both from the source and the voltage amplifier, and yields a sensitivity for $P_{3} / P_{1}$ in the range of $10^{-7}$. Our experiment is described in full details in Refs. $[11,12]$. The results reported here are for glycerol, an archetypical glass former with $T_{g} \simeq 190 \mathrm{~K}$.

3 Results and discussion This high sensitivity setup was used to test the predictions of Ref. [10] which, as evoked in the introduction, gives a relation between $\chi_{3}$ and the $T$ dependent average number of dynamically molecules $N_{\text {corr }}(T)$ through the following scaling form :

$$
\chi_{3}(\omega, T) \approx \frac{\epsilon_{0}\left(\Delta \chi_{1}\right)^{2} a^{3}}{k_{B} T} N_{c o r r}(T) \mathcal{H}(\omega \tau),
$$

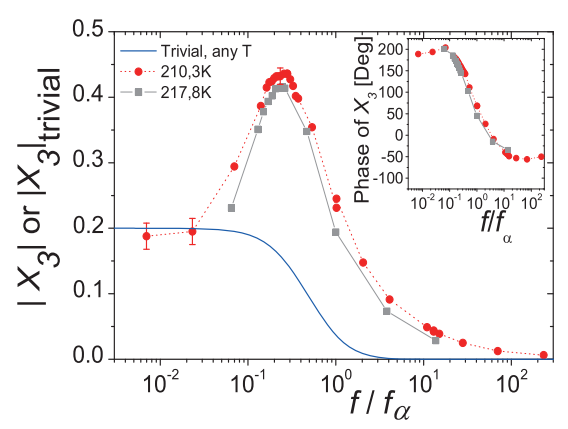

Figure 1 (Color online) For glycerol, the modulus of the dimensionless nonlinear susceptibility $X_{3}(\omega, T)=\chi_{3}(\omega, T) \times$ $k_{B} T /\left[\left(\Delta \chi_{1}\right)^{2} a^{3} \epsilon_{0}\right]$ is shown at two temperatures in the supercooled regime. For comparison, the corresponding quantity for independent polar molecules, labelled $\left|X_{3}\right|_{\text {trivial }}$, is plotted as a solid line (which does not depend on $T$ ). Both the humped shape of $\chi_{3}$ versus frequency, as well as its $T$ dependence, are distinctive features of glassy correlations. Inset: phase of $X_{3}$ (same symbols, phase of $X_{3, \text { trivial }}$ not shown).

where $\Delta \chi_{1}=\chi_{1}(\omega=0)-\chi_{1}(\omega \rightarrow \infty)$ is the part of the static linear susceptibility corresponding to the slow relaxation process we consider, $a^{3}$ the volume occupied by one molecule, and $\mathcal{H}$ a certain complex scaling function that goes to zero both for small and large arguments. This humped shape of $\mathcal{H}$ comes out due to glassy correlations [10]. To emphasize this point, let us consider the case of independent polar molecules undergoing (non inertial) rotational Brownian rotation. Their nonlinear dielectric susceptibility is given (see $[12,13]$ ) by the first prefactor of the right hand side of Eq. (1) times a function of $\omega \tau$ which has two main features: $i)$ it reaches its maximum value at $\omega=0$, ii) it does not depend on $T$, once plotted as a function of $\omega \tau$. This is why, in the following, we shall divide $\chi_{3}$ by the prefactor of the right hand side of Eq. (1), i.e. we shall focus onto the dimensionless nonlinear susceptibility $X_{3}(\omega, T)=\chi_{3}(\omega, T) \times$ $k_{B} T /\left[\left(\Delta \chi_{1}\right)^{2} a^{3} \epsilon_{0}\right]$. If Eq. (1) is relevant for supercooled liquids, one has $X_{3}(\omega, T) \approx N_{\text {corr }}(T) \mathcal{H}(\omega \tau)$. On the contrary, for non-interacting molecules, $\left|X_{3}\right|$ is a steadily decreasing function of $\omega \tau$ and does not depend on $T$. To illustrate this point, we have used the analytical results of Déjardin et al. [13], and plotted, on Fig. 1, the values of $\left|X_{3}\right|_{\text {trivial }}$ for independent molecules as a continuous solid line. Here the label "trivial" emphasizes the fact that the solid line on Fig. 1 has nothing to do with glassy correlations.

In Fig. 1, two sets of data for glycerol are given, corresponding to $T=210.2 \mathrm{~K}$ and to $T=217.8 \mathrm{~K}$, to illustrate the main results of Ref. [14] where much more data are reported. First, as predicted in Ref. [10], one observes on Fig. 1 that $\left|X_{3}\right|$ is "humped". Following Eq. (1), this humped shape comes from the transient nature of the 


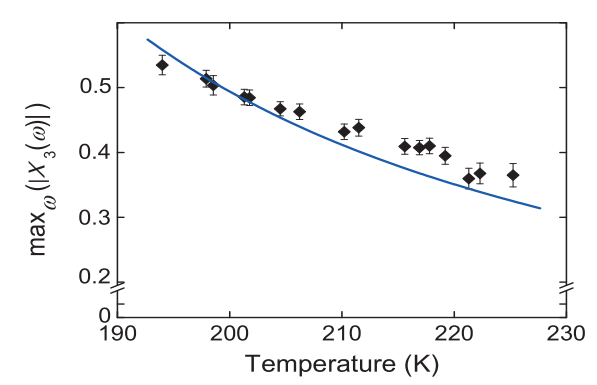

Figure 2 (Color online) For glycerol, the maximum value $X_{3, \max }$ of the modulus of $X_{3}(\omega)$ over frequency is plotted as a function of $T$. Its increase when $T$ decreases is interpreted as reflecting directly that of $N_{\text {corr }}(T)$ (see text). The solid line is the $T$ dependence of $T \chi_{T}$ with an arbitrary normalisation at 202 $\mathrm{K}$ (see text).

glassy correlations, the lifetime of which is of the order of $\sim 1 / f_{\alpha}$ : for $f / f_{\alpha} \gg 1$, the glassy correlations are not yet established, while for $f / f_{\alpha} \ll 1$ the liquid "flows", which destroys the glassy correlations, leaving only the "trivial" correlations above mentioned. This is why it was anticipated in [10] that the maximum of $\left|X_{3}\right|$ should arise for a frequency $f^{\star}$ of the order of $f_{\alpha}$; and Fig. 1 shows indeed that $f^{\star} \simeq 0.21 f_{\alpha}$. Furthermore, $\left|X_{3}\right|$ decays as a power law when $f \geq f_{\alpha}$ as predicted in Ref. [15], this point is illustrated in Fig. 2 of Ref. [14]. Last, defining, for each considered $T, X_{3, \max }(T)$ as the maximum of $\left|X_{3}(\omega, T)\right|$ over frequency, one observes that $\left|X_{3}(\omega, T) / X_{3, \max }(T)\right|$ falls onto a unique master curve, depending only on $f / f_{\alpha}$, in agreement with the prediction of Eq. (1). This scaling property can be guessed from the main part of Fig. 1, see also Fig. 2 of Ref. [14], as well as from the inset where it is shown that the phase of $X_{3}$ falls onto a $T$-independent master curve. Departures from this scaling occur at very low frequencies and/or at the highest temperature of $225 \mathrm{~K}$ reported in Ref. [14]. This is not surprising since in these two limits the role of the trivial nonlinearities evoked above is expected to contribute significantly to the measured $X_{3}$.

Having checked that the main predictions of Eq. (1) are relevant for the nonlinear susceptibility of glycerol, we now move to the $T$-dependence of $X_{3, \max }(T)$. As shown in Fig. 2, $X_{3, \max }$ increases when $T$ decreases. By using Eq. (1), we interpret this behavior as giving directly the temperature dependence of $N_{\text {corr }}(T)$ when $T$ decreases towards $T_{g}$. This is one of the main results of our study, and a strong experimental evidence of a growth of the dynamical correlation length close to $T_{g}$. To further support this interpretation, we plot on Fig. 2 the $T$ dependence of $T \chi_{T}$ defined by $T \chi_{T}=\max _{\omega}\left(T \partial\left(\chi_{\text {lin }}^{\prime}(\omega) / \Delta \chi_{1}\right) / \partial T\right)$. As explained in, e.g. Ref. [8], $T \chi_{T}$ should be proportional to $N_{\text {corr }}(T)$ up to an unknown prefactor proportional to $\chi_{0}$, where $\chi_{0}$ is related to the correlation between the lo-

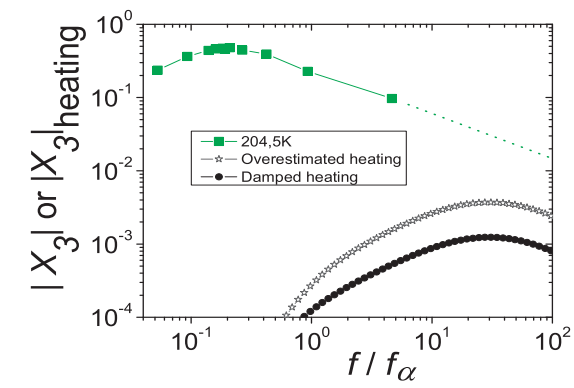

Figure 3 (Color online) For glycerol $\left|X_{3}\right|$ is compared at $T=$ $204.5 \mathrm{~K}$ to the heating contribution arising from the modulation of temperature at the frequency $2 \omega$ coming from the dissipation of electrical power in the supercooled liquid. The difference between the overestimated and damped heating contribution is explained in the text and in Ref. [12]. The heating contribution is in any case negligible with respect to the values of $\left|X_{3}\right|$ measured in glycerol. To ease the comparison with heating at very large values of $f / f_{\alpha}$, an extrapolation of the data of glycerol is plotted as a dotted line, following the power law behaviour reported in Ref. [14] at $210.2 \mathrm{~K}$.

cal fluctuation of enthalpy and the orientation of molecules [16]. If the amplitude of this "enthalpy-orientations" correlation is $T$-independent, then $T \chi_{T}$ captures the variation of the range of this correlation with $T$, and $\chi_{0}$ is $T$ independent. In that case, $T \chi_{T}$ is a fair estimator of $N_{c o r r}$, up to an unknown constant prefactor [16]. Assuming that $\chi_{0}$ does not depend on $T$ for Glycerol, one can compare the $T$-dependence of $T \chi_{T}$ and of $X_{3, \max }$. In Fig. 2, one sees that the $T$-dependence of both quantities are close (the unknown prefactor was used to make $T \chi_{T}$ coincide with $X_{3, \max }$ arbitrarily at $202 \mathrm{~K}$ ). This shows that the simpler method, introduced in [7] and used extensively in [9], where $N_{\text {corr }}(T)$ is drawn from $T \chi_{T}$ is valid at least for glycerol.

Expanding on the arguments given in Refs. $[12,14]$, we now comment on the possible contribution of the heating of the liquid to our third harmonics measurements. This heating comes simply from the fact that the strong applied electric field leads to some dissipated electrical power $p$ with a d.c. component as well as a component $p_{2}(t)$ oscillating at $2 \omega$. Before being absorbed by the thermal reservoir setting the base temperature $T$ of the experiment (hereafter represented by the metallic electrodes), this electrical power has to travel across the sample of thickness $e$. As a result a small temperature increase arises, containing a $2 \omega$ component $\delta T_{2} \sim p_{2}$. By using, as an order of magnitude estimate $\delta P(t) \simeq\left(\partial P_{\text {lin }}(t) / \partial T\right) \delta T(t)$, which involves a product of two terms oscillating at $\omega$ and $2 \omega$, one sees that a spurious contribution to the measured $P_{3}$ arises from $\delta T_{2}(t)$, i.e. from a part of the heating phenomenon.

In order to estimate this spurious effect, we have to compute $\delta T_{2}(t)$ for a supercooled liquid of total specific 
heat $c$, and thermal conductance $\kappa$. As shown in Ref. [17], the latter quantity does not depend on frequency, while the former depends on $f / f_{\alpha}$ due to the fact that the slow degrees of freedom relevant for the glass transition cannot equilibrate for frequencies larger than $f_{\alpha}$. The heat propagation equation for such a liquid surrounded by two thermal reservoirs (i.e. the electrodes) separated by the distance $e$, is solved by decomposing the heating into spatial modes of wave vector $K=m \pi / e$ with $m$ an odd integer. After spatially averaging the heating over the sample volume, one finds, keeping only the dominant $m=1$ spatial mode [12]:

$$
\delta T_{2}(t)=\frac{\epsilon_{0} \chi_{l i n}^{\prime \prime} \omega E^{2} e^{2}}{24 \kappa_{t h}} \frac{\cos \left(2 \omega t-\phi_{2}\right)}{\sqrt{1+\left(2 \omega \tau_{t h}\right)^{2}}}
$$

where $\phi=-\pi+2 \arctan \left(\chi_{1}^{\prime \prime} /\left(\chi_{1}^{\prime}-\chi_{1}^{\prime}(\infty)\right)\right), \tau_{t h}=$ $c e^{2} /\left(\kappa_{t h} \pi^{2}\right), \phi_{2}=\phi+\arctan \left(2 \omega \tau_{t h}\right)$ and $\epsilon_{0}$ is the dielectric permittivity of vacuum.

The simplest way to estimate the heating contribution $P_{3, h}$ is to set $P_{3, h}=\left(\partial P_{1}(t) / \partial T\right) \delta T_{2}(t)$, as evoked above. This clearly overestimates $P_{3, h}$ in the case $f \geq f_{\alpha}$ because $\delta T_{2}(t)$ oscillates at a frequency larger than the typical relaxation frequency $f_{\alpha}$ of the dipoles. A first estimate of the damping of the effect of $\delta T_{2}$ on the polarisation is given at the end of Ref. [12]. We shall not reproduce the equations here. We shall just illustrate the heating contribution by Fig. 3, where the overestimated and damped heating contributions to $\left|X_{3}\right|$ are plotted at $T=204.5 \mathrm{~K}$ and compared to the measured values of $\left|X_{3}\right|$ at the same temperature. The heating contribution is clearly negligible at any frequency. A detailed analysis (to be published elsewhere) shows that the heating contribution $\left|X_{3}\left(f / f_{\alpha}\right)\right|_{h}$ globally increases with $T$-contrarily to $\left|X_{3}\left(f / f_{\alpha}\right)\right|-$. The fact that heating is negligible at $204 \mathrm{~K}$ at any value of $f / f_{\alpha}$ thus guarantees that this is also true for any lower temperature. Last in the vicinity of $f^{\star} \simeq 0.21 f_{\alpha}$ where $\left|X_{3}\right|$ is maximum, the heating contribution is so small that it does not affect the data reported in Fig. 2, even at the highest temperature of $225.3 \mathrm{~K}$.

Let us emphasize that the heating that we have just discussed above must not be confused with that of the "inhomogeneous heating model" [18-20]. This "inhomogeneous heating model" intends to give a phenomenological description of the intrinsic nonlinear effects in supercooled liquids, and was shown to account for the nonlinear measurements at $\omega$ in, e.g., references [18-20]. In this framework, each dynamical heterogeneity has its own fictitious temperature on top of the temperature of the phonon bath. Some more work is needed to give a thorough comparison of this model at $3 \omega$ and our data.

4 Conclusion To summarize, we have discussed the behavior of the nonlinear susceptibility $\chi_{3}(\omega, T)$ of glycerol for a temperature interval from $T_{g}+4 \mathrm{~K}$ to $T_{g}+35 \mathrm{~K}$. The predictions of Eq. (1) have been shown to be relevant for the experimental data, and this is why we interpret the $T$ dependence of the height of the peak of $\chi_{3}$ as reflecting directly that of $N_{\text {corr }}(T)$. We think that any model aiming at describing the nonlinear susceptibility near the glass transition should be able to account for both the modulus and the phase of $X_{3}$ reported here. Indeed, these two quantities should contain important information about the dynamical correlations in the considered supercooled liquid. Furthermore accounting for the modulus and the phase of $X_{3}$ will allow to obtain the absolute value of $N_{\text {corr }}(T)$, which is not possible at present due to the unknown expression of the function $\mathcal{H}$. This is why, one can hope that a detailed understanding of the kind of data reported here will yield substantial progress in the modelisation of the glass transition, and perhaps to unveil its possible link with critical phenomena.

Acknowledgements We thank R. Tourbot for his outstanding technical help. We acknowledge interesting discussions with C. Alba-Simionesco, A. Lefèvre, R. Richert, M. Tarzia, and support by ANR grant DynHet.

\section{References}

[1] P.G. Debenedetti and F.H. Stillinger, Nature 410, 259 (2001).

[2] B. Schiener and R. Bohmer et al., Science 274, 752, (1996).

[3] U. Tracht et al., Phys. Rev. Lett. 81, 2127 (1998).

[4] R. Richert, J. Phys.: Condens. Matter 14, R703 (2002).

[5] M.D. Ediger, Annu. Rev. Phys. Chem. 51, 99 (2000).

[6] M.M. Hurley and P. Harowell, Phys. Rev. E 52, 1694 (1995); for a review see, e.g., W. Kob, arXiv:cond-mat/0212344v1.

[7] L. Berthier et al., Science 310, 1797 (2005).

[8] L. Berthier et al., J. Chem. Phys. 126, 184503 (2007).

[9] C. Dalle-Ferrier et al., Phys. Rev. E 76, 041510 (2007).

[10] J.-P. Bouchaud and G. Biroli, Phys. Rev. B 72, 064204 (2005).

[11] C. Thibierge, D. L'Hôte, F. Ladieu, and R. Tourbot, Rev. Sci. Instrum. 79, 103905 (2008).

[12] C. Crauste-Thibierge, C. Brun, F. Ladieu, D. L'Hôte, G. Biroli, and J.-P. Bouchaud, EPAPS of Ref. [14], available at http://prl.aps.org/epaps/PRL/v104/i16/e165703/CrausteEPAPS-modified.pdf.

[13] J.L. Déjardin and Yu.P. Kalmykov, Phys. Rev. E 61, 1211 (2000).

[14] C. Crauste-Thibierge, C. Brun, F. Ladieu, D. L'Hôte, G. Biroli, and J.-P. Bouchaud, Phys. Rev. Lett. 104, 165703 (2010).

[15] M. Tarzia, G. Biroli, A. Lefèvre, and J.-P. Bouchaud, J. Chem. Phys. 132, 054501 (2010).

[16] See the discussion on the possible $T$ dependence of the prefactor $\chi_{0}$ following Eq. (18) of [8]. Another caveat, still to be understood, is that for strong liquids one finds $T \chi_{T} \sim 1 / T$, instead of a constant value that one naively expects.

[17] N. O. Birge Phys. Rev. B 34, 1631 (1986).

[18] R. Richert and S. Weinstein, Phys. Rev. Lett. 97, 095703 (2006).

[19] S. Weinstein and R. Richert, Phys. Rev. B 75, 064302 (2007).

[20] W. Huang and R. Richert, Eur. Phys. J. B 66, 217 (2008). 